\title{
Literarisch-linguistische Besonderheiten in Wolfgang Hildesheimers Selbst-Übersetzungen*
}

\author{
Lucia Salvato (Milano)
}

\begin{abstract}
This paper presents a linguistic analysis of a lesser known part of the written production of German writer and artist Wolfgang Hildesheimer (1916-1991). The insight into his two speeches, delivered in English and self-translated into German, has two aims. First, to analyze their most peculiar linguistic features. Second, to highlight Hildesheimer's linguistic awareness and his extensive activity with languages and with their variations. Thanks to his bilingualism, he used to explore language variations and often realized some in a very peculiar way. The features highlighted in the analysis concern above all literal equivalents, the translation of foreign and loan words, of metaphors and idioms. This aims to emphasize Hildesheimer's ability to deal with languages and their qualities.
\end{abstract}

\section{$1 \quad$ Einleitung}

Wolfgang Hildesheimer (Hamburg 1916; Poschiavo/Schweiz 1991) ist als Schriftsteller und Künstler weltbekannt. Als Schriftsteller ist er hauptsächlich für seine Prosawerke berühmt, unter denen zunächst eine erste Sammlung von Kurzgeschichten, Lieblose Legenden (1952), zu erwähnen ist. Besonders beliebt sind seine zwei biographischen Werke, Mozart (1977) und Marbot (1981), von denen ersteres in mehr als zehn Sprachen übersetzt wurde und weltweit in zahlreichen Auflagen erschien. Aber obwohl auch seine Theaterstücke und Hörspiele bekannt sind, ist sein Ruhm als Schriftsteller vor allem an seine drei Prosatexte geknüpft, die in verschiedene Sprachen übersetzt wurden: Paradies der falschen Vögel (1953), Tynset (1965) und Masante (1973).

Innerhalb seiner reichen schriftstellerischen Produktion, für die er viel Anerkennung bekam, ${ }^{1}$ sind zwar seine Selbstübersetzungen weniger bekannt und doch voll bemerkenswerter

\footnotetext{
* Für Ermutigungen und Hinweise jeglicher Art möchte ich Hildesheimers Verwandten Inge und Monica Thurner sowie der Kollegin Serena Grazzini aus der Universität Pisa danken, die mit ihren Kommentaren und Hinweisen zur Verbesserung der Qualität des vorliegenden Artikels beigetragen haben. Weiterhin danke ich den zwei anonymen Gutachtern für sorgsames Lesen und wertvolle Anmerkungen.

${ }^{1} \mathrm{Zu}$ den Auszeichnungen und Ehrungen, die er für seine so produktive Karriere bekam, zählen der Hörspielpreis der Kriegsblinden für Prinzessin Turandot (1955), der Georg-Büchner-Preis und der Bremer Literaturpreis für Tynset (1966), der Große Literaturpreis der Bayerischen Akademie der Schönen Künste (1982), das Große Bundesverdienstkreuz der Bundesrepublik Deutschland (1983) und der Weilheimer Literaturpreis (1991).
} 
sprachlich-linguistischer Besonderheiten. Die vorliegende Arbeit beschäftigt sich mit zwei Texten, nämlich zwei englischen und ins Deutsche übersetzten Reden, die sein lebhaftes Interesse an Sprachen bezeugen. Zur Entstehung dieses Interesses trugen sicher einige postschulische Tätigkeiten während des zweiten Weltkriegs ${ }^{2}$ im Ausland bei, wo er die englische Sprache perfektionierte und bald als zweisprachig erkannt wurde. Überdies bekam er nach dem Krieg eine dreijährige Stelle als Simultandolmetscher bei den Nürnberger Prozessen, eine Tätigkeit, die ihm auch für seine spätere übersetzerische Aktivität wertvoll wurde.

Da nun Hildesheimers Interesse an Sprachen auch und vor allem auf seinen eingehenden Kenntnissen in deutscher und englischer Sprache beruht, werde ich im nächsten Abschnitt von seinem Bilingualismus ausgehen und anhand einiger Beispiele eine Einführung in die wesentlichen Merkmale seines Sprachgebrauchs geben. Abschnitt 3 ist Hildesheimers Übersetzungstätigkeit gewidmet: Seine Erfahrungen als Übersetzer betreffen vor allem die Übertragung englischsprachiger Literatur ins Deutsche und dienen hier als Einführung in seine kurze und doch nicht weniger interessante Erfahrung als Selbstübersetzer. Im letzten Abschnitt werden die Ergebnisse meines Fokus gesammelt dargelegt.

\section{$2 \quad$ Hildesheimers Bilingualismus}

Hildesheimers Erfahrung als Übersetzer ist mit seiner Karriere als Schriftsteller und offensichtlich mit seinem Bilingualismus in Deutsch und Englisch verknüpft. Sowohl seine schulische Ausbildung als auch seine ersten beruflichen Tätigkeiten zwischen Europa und dem Mittleren Osten ermöglichten ihm, seine Zweisprachigkeit zu vervollkommnen.

Aufgrund seiner Erfahrungen in jugendlichen Jahren zwischen Deutschland und England ${ }^{3}$ kann man bei ihm von einem successive bilingualism (,sukzessive Zweisprachigkeit'), einer Art ,aufeinanderfolgender Zweisprachigkeit" sprechen, nach der eine L1 (deutsch für ihn) von Geburt an als Erstsprache erworben wird und eine L2 (englisch für ihn) erst später als Zweitsprache folgt (cf. u. a. Bhatia/Ritchie 2004: 103-110; 114-120 und Hammer 1999). Wegen dieser „späten“ Begegnung mit der englischen Sprache kann man jedoch genauer von einem late bilingualism (,Zweisprachigkeit im Erwachsenenalter') sprechen, nach der eine L2 nach 16/17 Jahren erworben wird (cf. Bhatia/Ritchie 2004: 118 und Hammer 1999: 17-24).

\subsection{Hildesheimer und die englische Sprache}

Vor dem Ende der 1940er Jahre hatte sich Hildesheimer schon sehr intensiv mit der englischen Sprache befasst (cf. Braese 2016: 97-100; Jehle 2003: 403f.). Wie bereits erwähnt (siehe Fußnote 2), ermöglichten ihm seine guten Kenntnisse dieser Sprache, auch bedeutende Tätigkeiten

\footnotetext{
${ }^{2}$ Nach dem humanistischen Gymnasium in Mannheim (1926-1930) und der Odenwaldschule in Ober-Hambach (1930-1933) besuchte Hildesheimer für ein Jahr die Frensham Heights School in Farnham/England. Mit 18 Jahren fing er eine dreijährige Tischlerlehre in Palästina an, nach welcher er Malerei und Bühnenbildnerei in London studierte, und mit 24 Jahren begann er als Englischlehrer im British Council am Standort Tel Aviv und als Mitglied der britischen Administration zu arbeiten; dazu wurde er 1943 als Informationsoffizier in das British Public Information Office im Dienst der britischen Mandats-Regierung in Jerusalem aufgenommen; cf. Braese (2016: 108113) und cf. Hildesheimer (1993: 17-25).

3 Über die frühen Jahrzehnte Hildesheimers ist heute auch dank zweier im Jahr 2016 entstandener und von Stephan Braese und Volker Jehle geschriebener biographischer Werke ziemlich viel bekannt.
}

ISSN 1615-3014 
in englischsprachigen Ländern bzw. im Dienst der britischen Mandats-Regierung in den Jahren des zweiten Weltkrieges aufzunehmen. Zu dieser Zeit hatte sich folglich für ihn der Zugang zu einem multiethnischen Milieu eröffnet, das es so nur im Palästina der Mandatsjahre gab und für seine künftige künstlerische Arbeit unzweifelhaft eine prägende Bedeutung hatte. ${ }^{4}$

Dass Hildesheimer sich bis Ende der vierziger Jahre heimisch in der englischen Sprache fühlte („Damals sprach und schrieb er Englisch besser als Deutsch“, Jehle 2003: 403), zeigen nicht nur die zahlreichen Anglizismen in den frühen Lieblosen Legenden und die Briefe an seine Eltern (cf. Jehle 2003: 403), sondern auch die Anerkennung, die er für seine Worte in der englischen Ausgabe seiner deutschen Biographie Marbot (1981) erhielt. Es ist deswegen kein Zufall, dass seine ,erste nachweisbare literarische Veröffentlichung“ das Gedicht „Perpetuum Mobile“ ist, dessen Verse er in englischer Sprache abfasste. Sein Textsubjekt ruft einem weiblichen Gegenüber ein melancholisches ,goodbye“ nach (Braese 2016: 104) und beweist sein positives Urteil über diese an Begriffen der Körperlichkeit überreiche Sprache:

Englisch ist - wer wüßte es nicht! - eine an Konkreta überreiche Sprache, und in ihrer Üppigkeit zu wühlen - dem konnte ich damals nicht widerstehen. Allein schon der Reichtum an Begriffen der Körperlichkeit möchte darauf hinweisen, daß die Engländer ein Volk von Sensualisten seien, was aber wohl die Mehrheit entrüstet von sich weisen würde.

(Hildesheimer 1985/1991: 215, Hervorheb. i. O.)

\subsection{Hildesheimer und die deutsche Sprache}

Obwohl Hildesheimer seine Kindheit in Deutschland verbracht hatte und hauptsächlich als deutscher Erstsprachler betrachtet wird, begann er erst die deutsche Sprache unter neuen, linguistisch merkwürdigen Aspekten zu betrachten, als er Ende der vierziger Jahre nach Deutschland zurückkehrte (cf. Braese 2016: 149-212) und sie mit anderen Sprachen, vor allem der englischen, zu vergleichen anfing. In diesem Vergleich erkannte er nicht nur die Unterschiede zwischen den Beiden, sondern auch den Reichtum und die Vorzüglichkeit des Deutschen:

Die deutsche Sprache bedeutet mir sehr viel. Ich habe sie eigentlich erst kennengelernt, als ich wieder nach Deutschland zurückkam und sie mit anderen Sprachen vergleichen konnte. Sie ist in abstractis [...] die reichste Sprache, die es überhaupt gibt, nicht in konkreten Dingen, da ist vielleicht Englisch noch reicher. Aber sie ist für mich der ideale Ausdruck.

(Hildesheimer 1993: 80f.)

Einen engeren Kontakt mit Deutsch und Englisch „,erlebte“ Hildesheimer zwischen 1947 und 1949 als Simultandolmetscher im Nürnberger Gerichtssaal (siehe Abschn. 3.1), wo er als „Court-Interpreter“ (Hildesheimer 1999: 15) vor der Aufgabe und der Pflicht stand, ,im Sekundentakt in die syntaktische und grammatikalische Ordnung der je akut gesprochenen Sätze so sehr einzudringen, dass ein schleuniger Transfer in die andere Sprache bewerkstelligt, die Differenz überwunden werden konnte“ (Braese 2016: 142). Im Gerichtssaal stellte seine Dolmetscheraufgabe den Kontakt zu der unaufhörlich erneuerten Differenz zwischen den Sprachen her. Diese „als exakte, ,scharfe', immer neu spezifizierte, gleichsam ,ausdifferenzierte““

\footnotetext{
${ }^{4}$ Die Koexistenz von Juden, Arabern und Briten schloss einen interkulturellen Austausch ein, in dem eine Vielzahl weiterer Sprachkulturen wie auch der deutschen ihren Wert hatten. Doch blieb die englische Sprache die unumstrittene lingua franca, dank welcher die verschiedenen Kulturen bzw. unterschiedlichsten Menschen Kontakt zueinander aufnehmen konnten.
} 
(Braese 2016: 142) Ungleichheit wahrzunehmen, war die professionelle Pflicht der Dolmetscher, da sich das Gegenüber zwischen Richtern und Tätern für die Simultandolmetscher gerade in dieser Differenz realisierte. ${ }^{5}$

Aufgrund dieser zwar verlängerten intensiven und doch ersten zweisprachigen Tätigkeit behielt die Dolmetscher-Erfahrung für seine künftige Arbeit als Schriftsteller und Übersetzer zweifellos prägende Kraft, weil sie für ihn ,eine folgenreiche Schulung für sein Gehör des Deutschen und seine schon bald darauf einsetzende Textarbeit in dieser Sprache“ blieb (Braese 2016: 142f.). In den folgenden Jahren wurden seine Kenntnisse der deutschen Sprache so tief, dass Deutsch seine Schreibsprache blieb und er gerne ihre Feinheiten mit denen anderer Sprachen auch der romanischen - verglich und immer wieder neue Überlegungen darüber anstellte (cf. Hildesheimer 1985/1991: 213-216).

\section{$3 \quad$ Hildesheimers Übersetzungserfahrungen}

Aufgrund Hildesheimers Bilingualismus und lebhaften Anteils an Sprachen zielen nächste Abschnitte darauf ab, die wesentlichen literarisch-linguistischen Merkmale aus seinen Selbstübersetzungen hervorzuheben. Um das Ziel angemessen zu erreichen, wird zunächst ein kurzer Blick auf sein Erlebnis als Übersetzer geworfen.

\subsection{Hildesheimer als Übersetzer}

Auch als Übersetzer beschäftigte sich Hildesheimer vornehmlich mit ,englisch-deutschen oder deutsch-englischen Übersetzungen“, da diese die zwei Sprachen waren, die er vollkommen beherrschte (Hildesheimer 1985/1991: 211). Außer seinen ersten zwei „Übersetzungsexperimenten" ${ }^{6}$ und der einzigen Leistung mit einem italienischen Theaterstück ${ }^{7}$ übertrug er ab den $1950 \mathrm{er}$ Jahren vor allem englischsprachige Literatur ins Deutsche, ${ }^{8}$ darunter auch einige Theaterstücke ${ }^{9}$ (cf. Salvato 2018: 64, Fußnote 12).

\footnotetext{
${ }^{5}$ Während der Prozesse hatte Hildesheimer sogar Zugang zu den Nürnberger Archiven, und nach Ende seiner Arbeit als Dolmetscher beschäftigte er sich als Gerichtsschreiber und Redakteur mit zwei Bänden der amerikanischen Ausgabe der Prozess-Protokolle; cf. Braese (2016: 140f.).

${ }^{6}$ Diese fallen in die letzten zwei Jahre vor Kriegsende, als er die Übertragung eines Gedichts aus Stefan Georges Gedichtband Das Jahr der Seele (1897) und die Übersetzung von Kafkas Erzählung Elf Söhne (1916) veröffentlichte.

${ }^{7}$ Er entschied sogar, sich mit einem Theaterstück des italienischen Komödiendichters und Librettisten Carlo Goldoni zu beschäftigen, das er aus dem Italienischen (Un curioso accidente, 1760) ins Deutsche (Die Schwiegerväter, 1961) frei durch eine erweiterte und gleichzeitig nivellierte Bearbeitung übertrug.

${ }^{8}$ Er übersetzte einen Teil aus Finnegans Wake (1923-39) von James Joyce, ein Prosastück von Samuel Beckett (As the Story was Told, 1966; dt. Wie die Geschichte erzählt wurde, 1973) und zwei Theaterstücke von George Bernard Shaw (Saint Joan, 1924; dt. Die heilige Johanna, 1966 und Arms and The Man, 1898/1930; dt. Helden, 1970). Trotzdem, unter den mehreren gelieferten übersetzerischen Arbeiten literarischer Texte ist die einzige Übersetzung stricto sensu jene von Djuna Barnes Roman Nightwood (1936), den er für die deutsche Version Nachtgewächs (1959) betitelte. Das großartige Unterfangen bezeichnete er als „eine ungeheuerliche Arbeit“, für die er sich etwa 20 Jahre „sehr große Mühe“ gab und „eine lange Korrespondenz mit der damals noch lebenden D. Barnes“ hatte (Hildesheimer 1993: 83).

${ }^{9}$ Zwei Theaterstücke sind von Richard Brinsley Sheridan (The School for Scandal, 1782; dt. Die Lästerschule, 1960 und The Rivals, 1775; dt. Rivalen, 1961) und eines von William Congreve (The Way of the World, 1700; dt.
} 
Trotz dieser Erfahrungen war das Übersetzen nicht Hildesheimers primärer Arbeitsbereich und von Beruf fühlte er sich nicht einmal ein Schriftsteller. Vielmehr - und wie er selbst in seiner Lausanner Rede feststellte - betrachtete er seine Übersetzungserfahrungen als „eine kurze Reihe von Zwischenspielen“, die den Zweck hatten, sich „,an anderen, besseren zu messen“ (Hildesheimer 1985/1991: 215; cf. Jehle 2003: 404).

Wie definiert aber Hildesheimer seine übersetzerischen Arbeiten?

Geht man zunächst von Roman Jakobsons triadischer Definition aus (Jakobson 1959: 233), fallen Hildesheimers Übersetzungen zwar unter die zweite Typologie der interlingual translation bzw. translation proper - alle betreffen vorwiegend die von ihm bestens beherrschten zwei Sprachen -, sie wurden jedoch mit dem Ausdruck „Hinübersetzungen“ (sic!) ${ }^{10}$ definiert.

Nicht alle seinen Übersetzungserfahrungen kann man jedoch auf die traditionelle interlinguale Typologie zurückführen, weil er keine spezifische „Äquivalenz"11 zwischen Ausgangstext (AT) und Zieltext (ZT) berücksichtigte. Die meisten seiner Arbeiten fallen unter andere Typologien von Textverarbeitung und werden als „Bearbeitungen“, „Nachdichtungen“ oder „Übertragungen" bezeichnet. ${ }^{12}$

Eine erste erwähnenswerte Form von Übersetzung ist die von ihm bezeichnete „deutsche Paraphrase“ vom achten und letzten Kapitel des ersten der vier Bücher von James Joyces Finnegans Wake (aus der endgültigen Fassung der Buchausgabe 1939). In der „Erläuterung, Analyse und Interpretation" der ersten 56 Zeilen (Hildesheimer 1969/1991: 338-351) kennzeichnet er seine Arbeit als „eine Demonstration der Unübersetzbarkeit“" von Joyces Werk; die Arbeit käme zwar „dem Original streckenweise nah“, und doch könne sie niemals „das originale Idiom wiedergeben, seine verblüffende und zwingende Genauigkeit, seine Schönheit“" (Hildesheimer 1969/1991: 347; cf. Salvato 2018: 71-76).

Unter den Formen von Übersetzung, die Hildesheimers Experimentieren einer nicht wörtlich originalgetreuen und doch wirkungstreuen Textverarbeitung beweisen, ist seine Übertragung des Gedichts von Stefan George „Trauervolle Nacht“ (1897), die in der englischen Version im Jahr 1944 unter dem Titel „In mournful night“ erschien. Dass es sich um eine freie Übersetzung bzw. Nachdichtung handelt, ist schon vom ersten Vers bestätigt, „In mournful night you heard“, in dem der Übersetzer Hildesheimer dieselbe Freiheit zeigt, „die der Dichter genießt“" (Jehle 2003: 402). Ein nachgedichtetes Werk ist zwar ein Verfahren der literarischen Textverarbeitung, das vor allem eine freie und doch wirkungstreue Gedichtübersetzung ist, bei der der

Der Lauf der Welt, 1982), von dem auch die Bildunterschriften in Ronald Searles Quo vadis? und sechs seiner Bildergeschichten (1962) sind.

${ }^{10}$ Mit „Hinübersetzung“ soll das „Hinüberspringen“ eines Textes von einer Sprache in die andere verstanden werden (cf. Duden 2011: 863). Zur Bestimmung des Begriffs cf. Kromann/Rieber/Rosbach (1984); cf. auch Cedillo (2004: 98-101); Jehle (2003: 403f.); Kromann (1989: 270).

${ }^{11} \mathrm{Zu}$ dem lang debattierten „Problem der Übersetzbarkeit“ und dem damit verbundenen „Äquivalenzbegriff“ cf. u. a. Koller (2011: 159-277).

12 In der sog. ,modernen Translationstheorie“ (Ammann 1990: 107) wird die Notwendigkeit einer sachlichen bzw. terminologischen Abgrenzung zwischen den verschiedenen Definitionen und Typologien von Textverarbeitung immer noch bestritten. Zu ihrer Definition und deren Unterschieden cf. insbesondere Schreiber $(1993 ; 2004)$ und cf. Duden (2011: 264, 1229, 1807); Duden Online Version; Hausmann (2005: XI-XX); Koller (2011: 8, 42f., 58, 76-79); Wilpert (2001). 
Übersetzer dem Originaltext weder beim Wortschatz noch beim Stil, sondern vorwiegend bei der Wirkung treu bleibt. Stellt man nämlich Georges Gedicht und Hildesheimers englische Version nebeneinander, fallen sofort lexikalisch-syntaktische Besonderheiten bzw. stilistische Unterschiede auf (cf. Salvato 2018: 65-66), die jedoch keine starken Veränderungen in der Ausdrucksstärke der Nachdichtung Hildesheimers bewirken. In seinen literarischen Textverarbeitungen legte nämlich Hildesheimer weniger auf die formale als vielmehr auf die inhaltliche Übereinstimmung zwischen dem AT und dem ZT Wert.

\subsection{Hildesheimer als Selbstübersetzer}

Die zwei Reden, die Hildesheimer ursprünglich in englischer Sprache für ein englisches Publikum dachte bzw. verfasste und folglich mit einer englisch-sprachigen Aufnahme rechnete, sind „The End of Fiction“ (1975/1991a) und „The Jewishness of Mr Bloom“ (1984/1991a). Beide Reden wurden dann in demselben Jahr ihrer Erscheinung für ein deutsches Publikum ins Deutsche mit den Titeln „Das Ende der Fiktionen“ (1975/1991b) und „Das Jüdische an Mr Bloom“ (1984/1991b) übersetzt:13 Dementsprechend kann man hier von einem Fall des Selbstübersetzens reden.

Mit Selbstübersetzung ${ }^{14}$ wird sowohl der Prozess als auch das Produkt einer Übersetzung bezeichnet (cf. Grutman 2001: 17; Grutman 2009: 257 und cf. u. a. Salmon 2013: 79), die von demselben Autor des Originaltextes gemacht wird. Seit den im 20. Jahrhundert intensiv betriebenen Forschungen auf dem Gebiet der Fremdübersetzung (cf. Hokenson/Munson 2007) haben auch Selbstübersetzungen die Aufmerksamkeit von Sprachwissenschaftlern und Übersetzungsforschern auf sich gezogen. Und dank der Veröffentlichung der Erstausgabe der Routledge Encyclopedia of Translation Studies (1998/2001) wurde sogar ihre Forschung als spezifischer Teilbereich der Translation Studies anerkannt.

Verschiedene Autoren aus der europäischen Literatur haben Selbstübersetzungen geliefert; unter ihnen sind die bekanntesten James Joyce, Samuel Beckett, Stefan George und Rainer Maria Rilke. Die Frage ist aber, warum einige Schriftsteller in einer zweiten Sprache wiederholen, was sie schon in einer vorigen Version formuliert haben. Einige können diese Tätigkeit als eine Herausforderung annehmen, ,um künstlerische und kulturelle Unterschiede in verschiedenen Sprachen auf unterschiedliche Weise darzustellen“ (Loserl 2011: 35); andere können von ideologischen wenn nicht sogar kommerziellen Gründen angeregt werden. ${ }^{15}$

Warum auch Hildesheimer eine deutsche Übersetzung von zwei eigenen und schon auf Englisch verfassten Vorträgen lieferte, wurde von ihm nicht eindeutig erklärt. Höchstwahr-

\footnotetext{
${ }^{13}$ Nach Jehle übersetzte Hildesheimer nicht, sondern er ,,̈̈bertrug“ die beiden Reden, denn sie gehören zu den „,schwierigen Texten', die er in der Dankrede vor der Bayerischen Akademie erwähnt“; Jehle (2003: 404).

${ }^{14}$ Im Deutschen werden auch die Termini Eigenübersetzung oder Ipsoübersetzung verwendet (cf. u. a. Chung 2013), während man im Englischen die Termini self-translation oder auto-translation im Gegensatz zu normal bzw. ordinary translation bevorzugt (cf. Toury 2012: 279, 282-284; Hokenson/Munson 2007: 12-14; Routledge Encyclopedia 1998/2001: 17); cf. dazu auch Salvato (2018: 62f.).

${ }^{15}$ Wie Simona Anselmi darauf hingewiesen hat, werden Autoren von einem verschiedenen „telos“ („,the personal and ultimate goal of a translator") zur Selbstübersetzung angeregt. Man spricht von „,editorial“, ,poetic“, ,ideological“ oder „economic or commercial reasons“, die auch mit den Stellungen des Übersetzers vom sog. „social strand" in Translation Studies fokussiert werden; cf. Anselmi (2012: 33-55) und cf. Chesterman (2009: 19).
} 
scheinlich blieb er aber immer unabhängig von marktbezogenen Erwägungen, denn er schrieb eher nicht wie viele andere ,to gain access to a greater readership“ bzw. wegen eines ,mercantile interest" (Anselmi 2012: 53) und er äußerte nie seine Unzufriedenheit mit vorhandenen Übersetzungen seiner Texte, denn er war ihrer Schwierigkeit sehr bewusst (cf. Hildesheimer 1993: 80). Mit Sicherheit weiß man aber, dass es sich für ihn beim Übersetzen um keine mühelose oder mechanische Tätigkeit handelte, „denn es gibt Dinge, die in allen Sprachen anders klingen“ und dagegen sei oft „nichts zu machen“ (Hildesheimer 1993: 80). Dementsprechend kann man seine Entscheidung, seine zwei englischen Reden ins Deutsche zu übersetzen, eher auf seine fast perfekte Zweisprachigkeit zurückführen.

Die Schwierigkeit des Übersetzens besteht nach Hildesheimer auch darin, dass übersetzbare Termini zwar im Lexikon gefunden, doch nicht rastergemäß eindeutig reproduziert werden können. Man solle deshalb ,nur aus Sprachen übersetzen, die man so beherrscht wie die eigene“, und dafür in Lexika nur dann nachschlagen, ,um festzustellen, daß man sie nicht braucht“ (Hildesheimer 1985/1991: 214). Dennoch könne die Übersetzung einer selbst geschriebenen Rede eine noch größere und anstrengendere Herausforderung, als die Übersetzung der Rede eines anderen sein. Nicht zufällig stellte Hildesheimer in der Vorbemerkung zur deutschen Version seines zweiten Vortrags fest, dass eine Rede „,nicht übersetzbar“ ist, ,und eine selbst gehaltene oder zu haltende schon ganz und gar nicht" (Hildesheimer 1984/1991b: 196). Vor der „Utopie der vollkommenen Vermittlung des Originals“ und somit der sowohl theoretisch als auch praktisch unmöglichen „,idealen Übersetzung“ (Koller 2011: 296, 133) haben immer die beiden Thesen der Unübersetbarkeit ${ }^{16}$ und Übersetzbarkeit ${ }^{17}$ sprachlicher Texte gestanden.

\footnotetext{
${ }^{16}$ Bekannt sind darüber verschiedene Sprachauffassungen, die sich in den Jahrzehnten letzten Jahrhunderts entwickelt haben; aus jenen von Trier (1931) und Weisgerber (1971), aber vor allem von Sapir $(1921 ; 1983)$ und Whorf (1956) folgten z. B. wesentliche Konsequenzen in der Übersetzbarkeitsproblematik. Trier und Weisgerber entwickelten die Lehre der einzelsprachlich unterschiedlich aufgebauten sprachlichen Felder, nach denen ,die Bedeutungen einzelner Wörter verschiedener Sprachen streng genommen nicht miteinander verglichen und schon gar nicht gleichgesetzt werden können“ (Koller 2011: 172; kursiv im Original); Sapir und Whorf entwickeln ihrerseits „das linguistische Relativitätsprinzip“, das „aus der Tatsache der Strukturverschiedenheit der Sprachen folgt“ und auf der These basiert, dass jede Sprache „ein eigenes riesiges Struktursystem“ ist, in welchem „die Formen und Kategorien kulturell vorbestimmt sind“ (Whorf 1956; dt. 1963: 20, 53). Sapir-Whorf-Hypothese hat tatsächlich mit der These der Gleichsetzung von Denken und Sprechen und folglich mit der „Determiniertheit der Wirklichkeitserfassung durch die Struktur der Sprachen“ zu tun (Koller 2011: 173). Aus solchen Sprachauffassungen ergeben sich für die Übersetzbarkeitsproblematik wesentliche Konsequenzen: Sprachen sollten ihrem Wesen nach unübersetzbar sein, denn die durch Übersetzungen transponierten sprachlichen Inhalte konstituieren ,unterschiedliche geistige Zwischenwelten“ (Koller 2011: 172), die infolgedessen schwer übersetzbar werden. Zu Kollers Erläuterung der „relativen Übersetzbarkeit“ in Verbindung mit dem „sprachlichen Relativitätsprinzip“ cf. Koller (2011: 170-180).

${ }^{17}$ Im Gegensatz zur These der Unübersetzbarkeit stehen sicher das „Axiom der Übersetzbarkeit“ und das „der Ausdrückbarkeit“" (Koller 2011: 185-190), die von Sprachwissenschaftlern wie Hjelmslev, Weinrich, Jakobson, Lenneberg befolgt wurden; aber auch die unbestrittene praktische Erfahrung von Übersetzungen und „das unbestreitbare Gelingen der Kommunikation" durch Übersetzungen auch zwischen strukturell stark verschiedenen Sprachen zählen dazu; diese Erfahrung hat in der Geschichte der Übersetzungspraxis bewiesen, dass die verschiedenen Sprachen im Wesentlichen flexibel, dynamisch und vielschichtig sind und haben eher keine letztlich statische Struktur, wie dagegen Weisgerbers und Whorfs Thesen zu beweisen versuchten; cf. Koller (2011: 174). Zu Kollers Begriffsbestimmung der „prinzipiellen Übersetzbarkeit“ cf. Koller (2011: 181-190).
} 
Wie wichtig ist aber für Hildesheimer die Texttreue? Hängt er an jedem Wort seiner Reden, oder ist er auch bereit, einige Wörter oder komplette Sätze abzuändern bzw. zu ersetzen oder sogar zu entfernen?

Sollte man sich an seine hier schon erwähnten Worte halten (siehe Hildesheimer 1993: 80; ders. 1984/1991b: 196), könnte man glauben, dass seine Reflexion nur die Unmöglichkeit einer echten Übersetzungserfahrung betrifft und wahrscheinlich deswegen wörtliche bzw. dem Original streng verbundene Übertragungen bevorzugte. Bei näherer Betrachtung sieht man jedoch, dass er in seinen Übersetzungserfahrungen auch bereit war, das Original auf eine neue bzw. auf seine eigene Form zu übertragen (siehe hier Abschn. 3.1 und insbesondere Fußnoten 7-10). Einige beispielhafte Textpassagen, in denen Hildesheimer seine eigenen Worte vom Englischen ins Deutsche übersetzt, sollen im Folgenden sowohl seine genaue Nachahmung des Originaltextes als auch seine Freiheit davon verdeutlichen.

Es sei zunächst betont, dass mehr als die Hälfte der analysierten Termini und Ausdrücke eine „Wörtliche“"Wiedergabe des Originals sind. Dies bestätigt sowohl Hildesheimers Bestreben, seine ursprünglichen Worte „mit größtmöglicher Genauigkeit““ (1984/1991b: 196) wiederzugeben, als auch die Feinheit, mit der er den treffenden Terminus suchte und oft auch fand. Die Textstellen aus beiden Reden bzw. Versionen werden hier hintereinander wiedergegeben, um ihre Unterschiede bzw. Übereinstimmungen und dadurch Hildesheimers Übersetzungsentscheidungen zu ermitteln. Sie betreffen vor allem vier Hauptbereiche: Wort-für-Wort Übertragungen, Fremd- und Lehnwörter, Metaphern und Redewendungen sowie Alliterationen. Da die meisten eine wörtliche Wiedergabe des Originals sind, werden zunächst ein Paar Beispiele von Hildesheimers Genauigkeit beim Übersetzen gezeigt.

\subsubsection{Wörtliche Entsprechungen}

Auf der Ebene der These der Übersetzbarkeit sind wortwörtliche Übersetzungen immer - insbesondere seit den Bibel-Übersetzungserfahrungen des Theologen und Kirchenvaters der Christenheit Hieronymus - ein wissenschaftlicher Kernpunkt innerhalb der Übersetzungsgeschichte (cf. u. a. Kollers Erläuterung von Luthers und Schleiermachers Thesen der Verdeutschung und Verfremdung von Übersetzungen; Koller 2011: 32-39). Jedenfalls glaubt man eher nicht, dass eine vollkommene Eins-zu-eins-Beziehung zwischen Formen und Inhalten sowohl bei intralingualen (innerhalb derselben Sprache) als auch bei interlingualen (zwischen zwei oder verschiedenen Sprachen) Übersetzungen bestehen kann. ${ }^{18}$

Anders aber als in der kontrastiven Linguistik, in der die regelhaften Korrelationen zwischen zwei Sprachsystemen systematisch bzw. nach einem bestimmten Grammatikmodell auf der Ebene der langue miteinander vergliechen werden (cf. dazu u. a. Ivir 2004 und Nickel 1980), untersucht die Übersetzungswissenschaft die Bedingungen der Übersetzungsäquivalenz und beschreibt die Zuordnungen von Äußerungen und Texten mit Bezug auf das parole-Sprachvorkommen (cf. u. a. Koller 2011: 220-226). Im Folgenden wird die Äquivalenzfrage primär unter

\footnotetext{
${ }^{18}$ Das ist der Grund, weshalb die Ergebnisse automatischer bzw. auf dem Wort-für-Wort Prinzip basierter Übersetzungsverfahren oft ungenügend und unbefriedigend vorkommen, da die Voraussetzung einer in der Sprach- und Textanalyse qualitativ befriedigende Übersetzung nicht immer erfüllt wird; cf. die Beispiele in Koller (2011: 72$75)$.
} 
sprachwissenschaftlichem Aspekt, d. h. unter Berücksichtigung sprachlich-stilistischer Aspekte und eher der spezifischen lexikalischen Entscheidungen Hildesheimers analysiert.

Die erste Gruppe von Zitaten betrifft die wörtlichen Übertragungen, in denen die deutsche Wort-für-Wort-Wiedergabe des ursprünglichen englischen Textes imitativer bzw. assoziativer Natur ist. Im ersten Beispiel kommen etwa Wörter gleichen Sprachstammes vor: ${ }^{19}$

What else - we ask ourselves - could that mean as that they have lost grip over their craft, having become insecure as to the value of fiction and its possibility of describing reality!

Bedeutet es, daß sie den Griff über ihr Handwerk verloren haben, daß sie unsicher über Wert und Gültigkeit der Fiktionen geworden sind?

In der englischen Version verwendet Hildesheimer ein ,phraseal verb“ (to lose grip) ${ }^{20}$ im Sinne von ,weniger effizient werden ' oder, weniger fähig sein', mit etwas umzugehen; in die deutsche Version überträgt er dagegen die beiden englischen Termini wort-wörtlich: Die Vergangeheitsform have lost des Verbs to lose (dt. verlieren) wird im Deutschen verloren haben und das Substantiv grip kommt als Griff vor. Dank der zweiten Lautverschiebung ist nämlich das deutsche Substantiv Griff die perfekte Entsprechung des englischen Wortes grip, das aus dem Germanischen stammt und sich zum neuhochdeutschen Wort mit der Gemination des stimmlosen labiodentalen frikativen Lautes [f] zu Griff umgewandelt hat (cf. Mackensen 2013: 171f., 179).

Weitere Beispiele einer lexikalischen Übereinstimmung der deutschen Version zum englischen Original sind die Ausdrücke, die in den beiden folgenden Textpassagen enthalten sind. Darin schließt Hildesheimer seine Rede ,in einer noch unakademischeren Weise“ und gibt die Worte eines imaginären Dialogs zwischen einem Wissenschaftler bzw. Genetiker (Engl. scientist) ,auf der Höhe seines Berufs“ und einem Romancier (Engl. novelist) ,,auf der Höhe des seinen“ wieder (EF 156):

SCIENTIST Anyway, it is too late to blame science. We cannot stop its development and therefore not its effect.

NOVELIST You see? Warning is part of our reality.

SCIENTIST That is how you see it. And what you make your reader believe. But it could be that only if you were ahead of us. Whereas in fact you are lagging behind.

(EF 139)

GENETIKER Wie auch immer: es ist zu spät, die Wissenschaft verantwortlich zu machen. Du kannst ihre Entwicklung nicht aufhalten, daher auch nicht ihre Wirkung.

ROMANCIER Das ahnen wir. Und die Ahnung ist ein wesentlicher Teil unserer Wirklichkeit. Sie bedingt die entsprechende Warnung.

\footnotetext{
${ }^{19}$ Hier und im Folgenden werden Passagen aus The End of Fiction/Das Ende der Fiktionen mit EF, die aus The Jewishness of Mr Bloom/Das Jüdische an Mr Bloom mit JB sowie jeweils der entsprechenden Seite gekennzeichnet. Sämtliche Hervorhebung in den Zitaten stammen von der Autorin des vorliegenden Beitrags.

${ }^{20}$ Der englische Ausdruck lose your grip bedeutet „to lose control over a situation“ und „If you lose your grip, you become less efficient and less confident, and less able to deal with things"; Collins Online English Dictionary (2020); Collins Cobuild (1987: 639; Hervorheb. im Original).
} 
GENETIKER Warnen könntet ihr nur, wenn ihr uns voraus wäret. In Wirklichkeit hinkt ihr hinterher.

(EF 157f.)

Für die Wiedergabe des ersten substantivierten Verbs - warning aus dem Verb to warn ${ }^{21}$ verwendet Hildesheimer zwar das genau entsprechende deutsche Derivat Warnung, im Sinne von das Warnen, das Gewarntwerden, also ein „Hinweis auf eine Gefahr“ oder „etwas, wodurch jmd. vor etwas gewarnt wird, werden soll“ (Duden 2011: 1970). Das deutsche Wort Warnung kommt aber erst am Ende des Satzes vor und dem lässt Hildesheimer ein anderes Wort nur im Deutschen vorankommen, nämlich das Wort Ahnung, und diese Hinzufügung soll ausdrücken, dass es Letztere - die Ahnung - ist, welche die entsprechende Warnung auslöst.

Daüber hinaus verwendet Hildesheimer in der englischen Passage auch ein phrase (,,ahead of“) und ein phraseal verb bzw. ein Idiom (,to lag behind“), denen zwei deutsche Ausdrücke als genaue Übersetzung beider englischer Termini entsprechen: Einerseits entspricht der Ausdruck jdm. voraus sein im Sinne von ,vor den andern, an der Spitze“ sein (Duden 2011: 1937) dem Englischen „were ahead of “22, andererseits heißt das Verb jdmn. hinterher hinken „schief gehen“, „,zurückbleiben“ und „hinter jmdm., hinter etw. hinkend hergehen“, „hinkend folgen“ (Duden 2003: 781; Duden 2011: 861), das gerade das englische to be ahead of 23 übersetzt.

Auch in beiden folgenden Passagen bemerkt man eine genaue Übereinstimmung zwischen der englischen und der deutschen Version, denn der englische Ausdruck unforseen catastrophy bleibt auch im Deutschen eine unvorhergesehene Katastrophe, genau wie das deutsche Derivat Fehlkalkulation dem englischen miscalculation entspricht. Der Grund dafür liegt in diesem Fall in dem gemeinsamen lateinischen Wortstamm:

NOVELIST You are the cynic and you are lagging behind. You sill take it for granted that there will be enough space on earth to sit down and read a book. Or vice versa: that we are not all estinguished by an unforseen catastrophy, a miscalculation of science.

(EF 140)

ROMANCIER $D u$ hinkst hinterher. Du gehst von der Annahme aus, daß es auf der Erde genug Platz geben wird, um sich hinzusetzen und ein Buch zu lesen. Oder umgekehrt: daß wir nicht alle ausgerottet werden, durch eine unvorhergesehene Katastrophe, eine Fehlkalkulation der Wissenschaft.

(EF 158)

Das englische Wort catastrophy und das deutsche Katastrophe, die im Sinne von „schweres Unglück" verwendet werden, stammen tatsächlich aus dem lateinischen catastropha, das aber seinerseits aus dem griechischen Wort katastrophé abgeleitet war (Duden 2011: 975); die

\footnotetext{
21 „If you warn someone about something such a possible danger or problem, you tell them about it so that they are aware of it"; Collins Online English Dictionary (2020); Collins Cobuild (1987: 1639; Hervorheb. im Original).

22 ,If you are ahead in your work or achievements, you have made more progress than you expected to and are performing well“; „If someone or something is ahead of another person or thing in relation to their work or achievements, they are more advanced or have made more progress“; Collins Online English Dictionary (2020); cf. Collins Cobuild (1987: 31; Hervorheb. im Original).

23 „If one thing or person lags behind another thing or person, their progress is slower than that of the other“; ,If you lag behind someone or something, you move or progress more slowly than them“; Collins Online English Dictionary (2020); Collins Cobuild (1987: 806; Fett im Original).
}

ISSN 1615-3014 
Basisform beider anderer Wörter, miscalculation und Fehlkalkulation im Sinne von „falscher Kalkulation“, stammt aus dem spätlateinischen Wort calculatio (,[Voraus]berechnung“, „Schätzung“, ,in Bezug auf etw. angestellte Überlegung, Schätzung“; Duden 2011: 584, 956)24. Diesbezüglich hätte Hildesheimer in seiner Übersetzung auch andere Termini eintragen können: Statt Katastrophe hätte er (schweres) Unglück oder dessen Synonyme wie Unglücksfall, Schicksalsschlag, Desaster, Misserfolg wenn nicht sogar Tragödie verwenden können (cf. Duden 2014: 548, 636, 950); statt Fehlkalkulation hätte er irrtümliche Annahme oder [Ein]schätzung, Berechnung sowie irrtümliches Kalkül wählen können (cf. Duden 2014: 542); er hat sich jedoch streng an das Original gehalten und sich lieber für seine genaue deutsche Entsprechung entschieden.

\subsubsection{Die Übertragung von Fremdwörtern und Lehnwörtern}

Hildesheimers englische und deutsche Worte der beiden Reden beweisen den wertvollen Einfluss, den der Wortschatz (fast) jeder Sprache durch das ständige Zusammentreffen mit anderen Sprachen erfährt und der sich in der großen Zahl immer neuer entlehnter Wörter oder Wortbestandteile ausdrückt.

Die Anwesenheit von Lehnwörtern sowie Fremdwörtern in einer Sprache beweist, dass es darin auch Wörter gibt, die auf eine verschiedene Weise bzw. nach unterschiedlichen Eigenschaften der Wortstruktur von den Regeln des Kernwortschatzes abweichen. Dies kann die Lautstruktur bzw. Aussprache, die morphologische Struktur sowie die Schreibung betreffen. ${ }^{25}$ Die auffällige Anwesenheit von Fremd- und Lehnwörtern in beiden Versionen von Hildesheimers Reden hebt darüber hinaus sowohl seinen Bilingualismus als auch seinen Anteil an Sprachen hervor, die sicher von seinem engen Kontakt mit anderen Kulturen motiviert waren, von denen er tatsächlich beeinflusst wurde (siehe hier Fußnote 2). Manchmal kommt das Fremdwort nur in einer Version vor und manchmal erscheint es dank der entsprechenden Übersetzung in beiden Texten; manchmal handelt es sich um ein Derivat, das eine direkte Übernahme etwa aus dem Französischen ist oder seinen Ursprung im Lateinischen oder im Griechischen hat.

Der Ausdruck nom de plume und das Adjektiv bourgeois sind z. B. zwei direkte Übernahmen aus dem Französischen und finden sich nur in der englischen Version; in der deutschen treten sie dagegen in ihrer entsprechenden Übersetzung - „Pseudonym“ und „bürgerlich“ - auf:

Its author called himself Palinurus, after the sea-captain in the "Aeneid" [...]. But the nom de plume was not meant to be a disguise. Behind it its author was intentionally recognizable: Cyrill Cannolly $[\ldots]$.

(EF 126)

\footnotetext{
${ }^{24}$ Zur linguistischen Herkunft beider Wörter siehe das deutsche Herkunftswörterbuch (Duden 2001: 380, 397) und das englische Online Etymology Dictionary.

25 Zur morphologischen und strukturellen Beschreibung der Fremwörter bezieht sich die Dudengrammatik (2016: 90f.) auf die traditionelle Unterscheidung nach zwei Hauptgruppen: Wörter als Ganzes mit dem aus einer anderen Sprache entlehnten Stamm (Bsp. Job, fit, Hotel, Balkon) und Wörter mit den aus anderen Sprachen entlehnten Bestandteilen, die nach bestimmten Regeln zu Wortstämmen kombiniert werden (Bsp. Polykondensat, bilateral, multikulturell). Zum Unterschied zwischen Fremdwort und Lehnwort cf. Bußmann (2008: 203f., 396).
} 
Sein Autor nannte sich Palinurus, nach dem Seekapitän aus der „Aeneis“ [...]. Doch galt das Pseudonym nicht der Tarnung. Der Autor gab sich zu erkennen: Cyril Cannolly [...].

(EF 142)

Bemerkenswert ist der Ausdruck nom de plume, der hier im bildungssprachlichen Gebrauch als französische Bezeichnung für „Schriftstellerdeckname“, „(Schreib)federname“, „Künstlername" verwendet wird: Er ist zwar ein französischer Ausdruck, er wurde jedoch seltsamerweise nicht im französischen, sondern im englischen Sprachraum geprägt, ${ }^{26}$ mit dem Hildesheimer eine lange Zeit im engen Kontakt geblieben war. Der Autor verwendet im Deutschen lieber das Wort Pseudonym, das aber seinerseits kein einheimisches, sondern ein entlehntes Wort aus dem Griechischen ist. ${ }^{27}$

Auch das ursprünglich französische Adjektiv bourgeois kommt nur in der englischen Version vor und dafür verwendet Hildesheimer in der deutschen das entsprechende einheimische Adjektiv bürgerlich:

The decline of fiction set in not only with the changing concepts as worked out at the Moskow congress $[\ldots]$, but also by the organic dying of the opposite, exactly that which the Soviet writers were up against: "bourgeois literature".

(EF 134)

Doch das Elend der Realismen hat nicht erst mit den Konzepten eingesetzt, wie sie vom Moskauer Kongress erarbeitet wurde [...], sondern mit dem organischen Absterben genau dessen, was der Kongreß vornehmlich bekämpfte: die „bürgerliche Literatur“.

(EF 151)

Verschieden sind auch die Termini, die aus der lateinischen Sprache stammen. Nicht immer werden sie jedoch in beiden Versionen verwendet; vor allem kommen sie nur in einer vor, während ihr Denotat in der anderen angewendet wird:

So she had not fallen for him but had picked him out of a number of equals.

Er hat sie also keineswegs betört, sondern sie hat ihn zum Primus inter pares gemacht.

Hier kommt der lateinische Ausdruck primus inter pares (,der Erste von mehreren im Rang auf der gleichen Stufe stehenden Personen“; Duden 2011: 1373) nur in der deutschen Version vor, in der ihn Hildesheimer als entlehnten Ausdruck mit groß geschriebenen erstem Wort verwendet.

\footnotetext{
26 „Nome de Plume (1823): ,Pseudonym used by a writer‘, literally ,pen name‘, is a phrase invented in English in imitation of nom de guerre"; Online Etymology Dictionary.

${ }^{27}$ Das deutsche Wort Pseudonym ist tatsächlich ein Lehnwort aus dem Griechischen $\psi \varepsilon v \delta \dot{\omega} v v \mu o \varsigma$ (pseudōnimos), wörtlich ,der Falschname', und besteht aus zwei Wörtern, nämlich $\psi \varepsilon \tilde{\delta} \delta o \varsigma$ (pseudos), die Lüge' aus dem verbalen

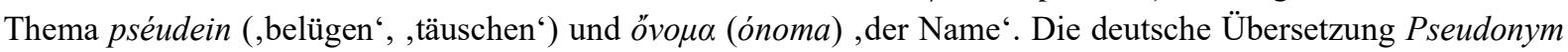
enthält ein Negationspräfix wie Miss- und Un- (z. B. Misserfolg, Unglück) oder auch Halbpräfix wie Schein-, Beinahe-, Quasi- (z. B. Scheinargument, Beinahelösung, Quasi-Automatismus), mit denen der Sprecher allgemein eine Taxierung ausdrückt, aber mit deren Gebrauch bei Spontanbildungen meistens eine kritische Wertung verbunden wird (cf. Duden 2016: 743f.; Weinrich 2007: 947-951). Zu seiner sprachlichen Herkunft cf. Duden (2001: 637).
} 
Auch in beiden folgenden Textpassagen aus beiden Versionen verwendet Hildesheimer zwei lateinische und doch großgeschriebene - also entlehnte - Fremdwörter:

"The supreme question about a work of art is out of how deep a life does it spring." I don't know to what point Joyce may have identified himself with his character in saying this [...] - but to me this seems to be one of the most pertinent statements about the work of art ever made $-[\ldots]$

„Das entscheidende Kriterium des Kunstwerks ist die Tiefe der Seele, der es entspringt.“ Nun weiß ich nicht, inwieweit Joyce sich hier mit dem Sprecher identifiziert [...] - aber für mich ist dieses Diktum eine der treffendsten Aussagen, die jemals über das Kunstwerk gemacht wurden $[\ldots]$

Above this drawing is the first line of the 'Odyssey', written in Greek. If the writing is Joyce's own, he must have copied it somewhere, as his selftaught Greek would not have allowed for this fluency of lettering:

Über der Zeichnung steht die erste Zeile der ,Odyssee‘. Wenn die Handschrift Joyces eigene ist, muß er die Zeile kopiert haben, denn sein geringes selbsterlerntes Griechisch hätte ihm diesen flüssigen Duktus wohl kaum gestattet:

Es geht um das zur Bildungssprache gehörende Wort Diktum aus dem Lateinischen dictum (,das Gesagte' als passive Form vom Partizip Perfekt; cf. Duden 2011: 422) und das aus dem Lateinischen entnommene Wort Duktus im Sinne von ,charakteristischer Art“ oder „,bestimmter Linienführung einer Schrift“" (,das Ziehen“, die ,Führung', ein ,innerer Zusammenhang '). ${ }^{28}$ Beide werden nur in der deutschen Übersetzung als entsprechende Form des im Original englischen Pronomens this und des Ausdrucks this fluency of lettering verwendet. Dazu kommt auch das

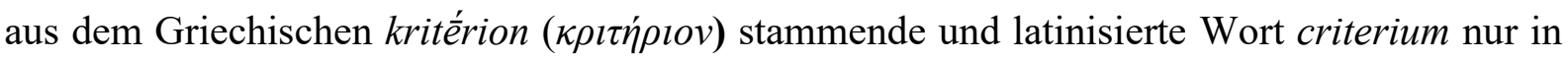
der deutschen Version vor. Es übersetzt das englische Wort question und kennzeichnet bildungssprachlich ein „unterscheidendes Merkmal als Bedingung für einen Sachverhalt, ein Urteil, eine Entscheidung“ (Duden 2014: 490; Duden 2011: 1063; Duden Online Version).

Quasi als Ausnahme werden dagegen zwei lateinische Ausdrücke in beiden Versionen verwendet und im bildungssprachlichen Gebrauch klein geschrieben behalten: ad nauseam (,bis zum Überdruss') und ad libitum (,nach Belieben'):

All possible thematic positions and combinations of the novels have been exercized ad nauseam, its possibilities of reproducing reality are exhausted [...].

\footnotetext{
28 In der Sprachwissenschaft wird der Duktus als ein bestimmtes Kennzeichen sowohl des Sprechens als auch des Schreibens verstanden und bezeichnet somit den charakteristischen Sprach- oder Schreibstil einer Person. Beim Sprechen werden sicher stilistische Elemente wie die Wortwahl und der Satzbau betrachtet; aber vor allem sind die Aussprache, Intonation und andere Spracheigenschaften der Prosodie, wie z. B. die Dialekteinflüsse, ein wesentliches Merkmal des Duktus eines Sprechers. Beim Schreiben bezieht sich der Duktus auf den Schreibstil einer persönlichen Handschrift, sehr oft eines Schriftstellers und seiner Art zu schreiben; diesbezüglich ist es möglich, Persönlichkeiten an ihrer Art zu schreiben, nämlich an ihrem Duktus zu identifizieren, wie es einigen Schriftstellern schon geschehen ist (cf. Duden 1997: 182, 336; Duden 2011: 449 und Duden Online Version).
} 
[...] die Möglichkeiten seiner Fabeln sind ad nauseam durchexerziert, seine Schauplätze und Akteure erschöpft.

(EF 149)

Its [of the bourgeois literature] great exponent was Thomas Mann [...]. A novelist who would depict and dispose of his subjects ad libitum, taken from any epoch, from biblical times until the beginning of this century $[\ldots]$.

(EF 134)

Ihr [der bürgerlichen Literatur] großer Vertreter war Thomas Mann [...] ein Romancier, der, in Verachtung allen Zeitbezugs, seine Geschichten ad libitum [...] auslas:

(EF 152)

Auch Entlehnungen aus dem Griechischen sind unter Hildesheimers Worten zu finden und auch darin gelten die gewöhnlichen Regeln der deutschen Sprache: Statt durch die Entsprechungen des lateinischen Alphabets werden z. B. die griechischen Buchstaben durch besondere Buchstaben und Buchstabenkombinationen dargestellt (cf. Duden 2016: 91); dies geschieht beispielsweise dem Konsonantgraphem 〈th〉 im Terminus Pathos aus dem Griechischen páthos ( $\pi \dot{\alpha} \theta o \varsigma$ : ,Schmerz', ,Leiden', ,Leidenschaft', ,das Ergriffensein“; cf. Duden 1997: 1316; Duden 2014: 686) zu páschein (,erfahren', ,(er)leiden`; cf. Duden 2011: 1136; Duden Online Version), der im folgenden Textauszug nur in der deutschen Version und innerhalb eines hinzugefügten Schaltsatzes verwendet wird:

I have to quote Ezra Pound again [...]. He says: "The man who tries to express his time instead of himself is doomed." Being without a library I could not look up the exact wording, but I am certain that "doomed" is the word. It is with some reluctance that I share a belief with Ezra Pound, but I am convinced of the validity of this statement:

(EF 130)

Ich muß nochmals Ezra Pound erwähnen. In seinem Buch „motz el son“ sagt er: „Der Mensch, der seiner Zeit Ausdruck zu geben versucht, statt sich selber, ist dem Untergang geweiht.“ Ich bin nicht gern einer Meinung mit Ezra Pound, aber von der Wahrheit dieses Satzes bin ich, trotz des Pathos, überzeugt.

(EF 146f.)

Statt Pathos hätte Hildesheimer das deutsche Wort Leidenschaft verwenden können, unter dessen Synonyme Begeisterung, Engagement, Neigung, Verlangen sind (Duden 2014: 603f.); er hat sich dagegen für eine Entlehnung aus dem Griechischen entschieden.

Anstelle von Fremdwörtern verwendet manchmal Hildesheimer Derivata, die als Lehnwörter den deutschen Regeln folgen. Als freie gemischte Form entnimmt die Lehnübertragung ${ }^{29} \mathrm{Fu}$ turologie das erste Element Futur aus dem Lateinischen futurum und das zweite -logie aus dem Griechischen lógos (cf. Duden 2011: 657 und Duden Online Version):

I contend that our future is unknown to us; that futurology, far from being an empirical science, let alone a real one, is a farce $[\ldots]$

\footnotetext{
${ }^{29}$ Unter „Lehnübertragung“ (engl. loan translation) versteht man sowohl den Vorgang als auch das Ergebnis einer genauen Glied-für-Glied-Übersetzung eines fremdsprachlichen Ausdrucks in die eigene Sprache; cf. Bußmann (2008: 396).
} 
Ich behaupte, daß unsere Zukunft uns unbekannt ist. Daß Futurologie - jämmerliches Wort! -, weit entfernt von einer Wissenscahft, noch nicht einmal Empirie, eine Farce ist [...].

(EF 156)

Im englischen Original stehen die zwei Termini (future und futurology) dank der Alliteration des stimmlosen labiodentalen Reibelauts [f] in phonetischer Beziehung; außerdem kann man hier eine rhetorische Figur aus der Gruppe der Wortwiederholungen erkennen, und zwar die Anadiplose oder reduplicatio, die als besondere Abart der geminatio (cf. Lausberg 1960/1990: $\S \S 607-622)$ als unmittelbare Verdoppelung eines Wortes in dem darauffolgenden Satz erkennbar ist. Da aber Hildesheimer in der deutschen Version das aus dem Lateinischen futurum stammende Derivat Futurologie neben dem Terminus Zukunft gebraucht, kommt es zu keiner Verdoppelung: Diese hätte geschehen können, wenn er das einheimische Kompositum Zukunftsforschung gebraucht hätte. ${ }^{30}$

\subsubsection{Die Übersetzung von Metaphern und Redewendungen}

Kann man vor metaphorischen Bildern einer spezifischen Sprache bzw. Kultur eine Äquivalenzbeziehung zwischen dem Ausgangstext und dem Zieltext herstellen?

Analysiert man das Verhalten der Übersetzer, wenn sie sich den übersetzerischen Herausforderungen der verschiedenen rhetorischen Stilmittel, insbesondere der Metaphern-Typen stellen, bemerkt man große Unterschiede in den vielfältigen, lebendigen, manchmal auch verwirrenden Ergebnissen, zu denen Übersetzer auch wegen eines verschiedenen Begriffs der Übersetzungstreue gelangen können (cf. Koller 2011: 256-261). Aristoteles definierte als erster mit Autorität die Metapher und unterschied vier Metapherntypen ,je nach der Richtung der Bedeutungsübertragung": ob "von der Gattung auf die Art" oder umgekehrt, "von der Art auf die Art" oder "gemäß der Analogie" (cf. Weinrich 1980). Seit Broeck (1981) unterscheidet man eher drei Haupt-Metapherntypen: die lexikalisierten oder toten Metaphern haben ihre Einzigartigkeit verloren, sind Teil des Lexikons einer Sprache geworden und sind deshalb nur noch aus einem sprachhistorischen Gesichtspunkt als bildhaft erkennbar; die konventionalisierten Metaphern sind traditionell bzw. poetisch/literarisch durch die Werke der meisten Schriftsteller institutionalisiert worden; und die privaten oder kühnen Metaphern werden als autorenspezifisch eher individuell bzw. okkasionell gekennzeichnet.

Die schwierigsten Übersetzungsprobleme tauchen zwar bei lexikalisierten Metaphern auf, weil sie meistens einzelsprach- und kulturspezifisch sind, doch eventuelle Lücken können oft durch unterschiedliche Verfahren wie das Umschreiben, den Kommentar, die Definition geschlossen werden. Demgegenüber lassen sich auch verschiedene Übersetzungsverfahren unterscheiden, wobei es vorwiegend möglich ist, Analogien in der Zielsprache auch ohne Verlust der originellen Bildkraft zu konstruieren. Die drei meist angewandten Verfahren sind die Übersetzung stricto sensu, die Substitution und die Paraphrase; bei dem ersten wird das der originellen Metapher zugrunde liegende Bild auch in der Zielsprache wiedergegeben; in der Substitution wird das der originellen Metapher zugrunde liegende Bild in der Zielsprache durch ein anderes ersetzt; in der Paraphrase wird die originelle Metapher in der Zielsprache nicht metaphorisch übersetzt, sondern paraphrasiert.

\footnotetext{
${ }^{30}$ Zur deutschen Komposition cf. Eichinger (2000).
} 
Nicht zufällig wird die These der prinzipiellen Unübersetzbarkeit zwischen Sprachen vor allem an einzelnen sogenannten ,unübersetzbaren Wörtern“ demonstriert (Koller 2011: 178, Hervorheb. i. O.), die adäquat verstanden werden, wenn ihr Zusammenhang mit der entsprechenden Kultur erlebt werden kann. Wegen dieses kulturellen Zusammenhangs wird dieselbe These auch für Metaphern und metaphorische Ausdrücke verteidigt, für die Übersetzer nur dann eine Entsprechung finden können, wenn jene zum allgemeinen Kulturgut bzw. zur „Weltliteratur“ gehören (cf. Koller 2011: 257).

Das Deutsche verfügt über einen großen Bestand an Wortgruppen, die als sprachliche Fertigteile der Form nach eher unveränderlich bleiben und deren Gesamtbedeutung sich nicht - oder nur schwer - aus den Bedeutungen der Einzelwörter erschließbar ist. Durch solche sprachlichen Fertigteile stellt die deutsche Sprache - vor allem durch die Umgangssprache und in der alltäglichen Kommunikation - immer neue Wendungen und Redensarten her (cf. Duden 2013: Vorwort).

Wie schon in vorigen Arbeiten darauf hingewiesen wurde (cf. Salvato 2018: 69f.; Salvato 2019: 111), kommen zwar Metaphern und Redensarten auch in Hildesheimers Reden doch fast nie in beiden Versionen vor, weil sie seiner Meinung nach „schlechthin unübersetzbar“ sind und „auch die optimale Lösung das Original nicht wiedergeben kann“ (Hildesheimer 1985/1991: 211). Diesbezüglich vertritt Hildesheimer die Auffassung der Unübersetzbarkeit von Wendungen, Redensarten und Metaphern auch in der deutschen Version seiner zweiten Rede (cf. Hildesheimer 1984/1991b: 196), in deren Vorbemerkung er den ,artifiziellen Charakter“ der rhetorischen Figuren in Übersetzungen betont. Tropen und rhetorische Figuren sind tatsächlich nach Hildesheimer ein erstes Beispiel sprachlicher Elemente, die bei der Übertragung in eine andere Sprache ihren natürlichen Charakter verlieren, weil sie sich innerhalb einer spezifischen Kultur entwickeln und die Lebensform der Menschen jener Kultur widerspiegeln. Der Versuch, sie in eine andere Sprache bzw. Kultur zu übertragen, fordert deshalb den Übersetzer heraus, durch treffende Bilder aus der Zielkultur eine ähnliche Wirkung in der Zielsprache zu erzielen.

Aufgrund des meist erfolglosen Versuchs, literarische Kunstgriffe zu übersetzen, entzieht sich Hildesheimer dem Problem, indem er entweder einen englischen bildlichen Ausdruck bzw. eine lexikalisierte Metapher mit einem deutschen denotativen Terminus bzw. Ausdruck wiedergibt oder durch eine nur in die deutsche Version hinzufügte Metapher einen englischen denotativen Ausdruck übersetzt.

Im folgenden Satz verwendet er z. B. eine deutsche Redewendung - kein(en) Hehl aus etwas machen (Duden 2013: 335) -, die das englische Denotat ,to make a secret of something' übertragen soll:

He will always speak about himself, and I, for one, do not intend to make a secret of it.

(EF 125)

Er spricht immer über sich selbst, und ich, für mein Teil, mache keinen Hehl daraus

In Wahrheit verwendet Hildesheimer den englischen Ausdruck nicht nach dem gewöhnlichen Gebrauch - to make no secret of something (Collins Online English Dictionary 2020) -, in welchem die Negation sich auf das Substantiv secret bezieht und nach welchem sein Satz ,and I, for one, intend to make no secret of it" lauten würde. Lieber verwendet er eine eigene Form, 
in welcher die Negation sich eher auf das Verb intend des Hauptsatzes bezieht und von welcher die feste deutsche Redewendung keine vollkommene Entsprechung realisieren kann.

Dasselbe geschieht in anderen Sätzen, in denen wieder die denotative Bedeutung der englischen Version in einen bildlichen Ausdruck übertragen und oft in der Form einer Kollokation wiedergegeben wird (cf. Bußmann 2008: 345):

Bloom operates with names like Maimonides and Moses Mendelssohn.

Dann wird Bloom Maimonides und Moses Mendelssohn ins Spiel bringen.

Die deutsche Redewendung kommt in diesem Fall in der Form eines Funktionsverbgefüges vor (jmdn./etwas ins Spiel bringen), das aus dem elementaren und daher relativ bedeutungsarmen Vorverb bringen und dem komplexen Nachverb bzw. Präpositionalobjekt ins Spiel besteht und somit die Bedeutung jmdn./etwas mit einbeziehen ausdrückt (Duden 2013: 699f.; cf. auch Duden 2011: 1642 und Duden Online Version). Es ist nämlich das Nachverb, das in seinem nominalen Bestandteil die wesentliche lexikalische Information des Funktionsverbs enthält. ${ }^{31}$

Oft kann aber auch das Gegenteil geschehen, nämlich dass ein bildlicher Ausdruck des englischen Originals in seine denotative deutsche Entsprechung übertagen wird. Für einige Ausdrücke verdeutlicht Hildesheimer lieber den Referenten, mit dem er wahrscheinlich die Unübersetzbarkeit zu vermeiden versucht. Dies geschieht z. B. mit dem Ausdruck mind or matter, der in der deutschen Version zwei Adjektiven entspricht:

$[\ldots]$ the function of even the most unexpected and trifling detail, mind or matter, never used in literature before, serves the purpose of building up a complete framework of physical reality.

(JB 183)

[...] daß hier die Funktion selbst des unerwarteten und trivialen Details, abstrakt oder konkret, wie es die Literatur zuvor nicht gekannt hat, der Absicht dient, ein möglichst lückenloses Gerüst physischer Realität aufzubauen.

Der englische bildliche Ausdruck mind or matter lehnt sich wahrscheinlich an einen anderen festen englischen Ausdruck, nämlich an das phrase bzw. expression „mind over matter“, das die Fähigkeit einer Person ausdrückt, „to control events, physical objects, or the condition of their own body using their mind" (Collins Cobuild 1987: 918; Collins Online English

\footnotetext{
31 Obwohl der Begriff „Funktionsverbgefüge“ (FVG) in der traditionellen Grammatik des Deutschen eine bedeutende Rolle spielt, ist heutzutage dafür noch keine klare, eindeutige Definition zu finden. Der Terminus bezeichnet zweiteilige Konstruktionen, die als verbale Gefüge eine inhaltliche Einheit darstellen und die Funktion eines Prädikats haben. Es geht deshalb um „feste“ oder ,,halbfeste“ Prädikatsausdrücke, die zwischen idiomatischen Verbindungen und Kollokationen angesiedelt werden. In einem FVG wie zur Diskussion stehen oder in Vergessenheit geraten ist z. B. das Nachverb kein einfacher Nominalausdruck, sondern ein Präpositional-Adjunkt, das meist ein deverbal abgeleitetes Nomen enthält. Diesbezüglich unterscheidet die Dudengrammatik zwei Varianten von FVG, die als Verbindung aus Funktionsverb und einerseits deverbalem Substantiv im Akkusativ (z. B. eine Erlaubnis geben), andererseits Portmanteau-Präposition mit deverbalem Substantiv (z. B. zur Anwendung bringen) vorkommen (cf. Duden 2016: 425-432; Weinrich 2007: 1053-1057; Winhart 2005).
} 
Dictionary 2020). Für seine deutsche Übersetzung verwendet dagegen Hildesheimer eine hochentwickelte Gegenüberstellung zweier sich widersprechender Begriffe, abstrakt und konkret.

Eine ähnliche Entscheidung trifft Hildesheimer vor einem anderen bildlichen Ausdruck, nämlich to gain the upper hand:

Because if the stream of consciousness always gains the upper hand.

Wenn nämlich der Bewußtseinsstrom immer dominiert.

Auch für die Übertragung dieses metaphorischen Originalausdrucks entscheidet sich Hildesheimer nicht für die deutsche Entsprechung - die Oberhand gewinnen - im Sinne von „,sich als stärker erweisen“, „sich gegen etw., jmdm. durchsetzen“ oder „die Führung haben/gewinnen“ oder „,bekommen/erhalten“ (cf. Duden 2013: 543; cf. Duden 2011: 1274 und Duden Online Version); er bevorzugt eher das einfache, denotative Verb dominieren, wegen dessen er folglich die metaphorische Färbung des Originals beiseitelässt.

Wie die hier dargebotene Analyse gezeigt hat, betreffen Übersetzungsprobleme in den meisten Fällen - nicht selten in Fach- und Sachtexten - den lexikalischen bzw. terminologischen Bereich. Insbesondere die literarische Sprache realisiert jedoch (fast) alle in der Sprache enthaltenen Möglichkeiten, welche auch die formal-ästhetischen bzw. spezifisch einzelsprachlichen Phänomene wie Reim, Alliteration, Sprachspiel, metrische Formen und Rhythmus betreffen und oft Assoziationen zu anderen Werken desselben Autors oder zu einer literarischen Tradition wecken. Auch Hildesheimers Werke dokumentieren seine breite Anwendung sprachlichstilistischer, insbesondere deutscher Merkmale, wie reimischer Formen, Alliteration, und nicht selten Sprachspiele, die in verschiedenen seiner vermischten Schriften enthalten sind und deshalb einer weiteren und ausführlichen Analyse wert wären.

\section{$4 \quad$ Schluss}

Die verhältnismäßig spät und quasi zufällig begonnene Schriftstellerkarriere Wolfgang Hildesheimers betrifft nicht nur weltberühmte Prosatexte, Theaterstücke und in viele Sprachen übersetzte biographische Werke. Als zweisprachiger Künstler, der in Deutschland, England und im britischen Mandatsgebiet Palästina gelebt hatte, verknüpfte Hildesheimer die Entwicklung seiner Karriere auch mit diversen, weniger bekannten Übersetzungserfahrungen.

Letztere wurden ohne Zweifel von seiner Zweisprachigkeit in Deutsch und Englisch gefördert, die auch seine Vorliebe für Sprachen und deren linguistische bzw. kulturelle Unterschiede prägte. Eine biographische Einführung (siehe Abschn. 1 und 2) hat deshalb den wesentlichen Aspekt von Hildesheimers Bilingualismus und analytischem Interesse an die möglichen Kombinationen der Sprache - besonders in der deutschen Lexik und Syntax - hervorgehoben.

Hildesheimers erste intensive Übersetzungserfahrung als Simultandolmetscher für Deutsch und Englisch sowie als Gerichtsschreiber und Redakteur bei den Nürnberger Prozessen (siehe Abschn. 2) war z. B. für ihn die Gelegenheit, eine gründliche bzw. folgenreiche Schulung für sein Gehör des Deutschen und seine zukünftige Arbeit auf diversen Texttypen zu erfahren. In den folgenden Jahren beschäftigte er sich nämlich als Übersetzer verschiedener literarischer Texte 
und ab den 1950er Jahren übertrug er vor allem englischsprachige Literatur - Theaterstücke und Prosatexte - ins Deutsche (siehe Abschn. 3.1). Weniger bekannt und doch linguistisch bemerkenswert ist diesbezüglich seine Tätigkeit als Selbstübersetzer (siehe Abschn. 3.2).

Das Ziel dieser wissenschaftlich-linguistischen Studie über Wolfgang Hildesheimer war deshalb, einige seiner weniger bekannten Texte zu analysieren, ihre wesentlichen sprachlich-linguistischen Merkmale hervorzuheben und somit seine umfangreiche Tätigkeit mit der Sprache und ihren Möglichkeiten aufzuzeigen.

Hinsichtlich seiner Übersetzungserfahrungen wurde in der Studie zunächst betont, dass er nicht nur wörtliche Übertragungen von Originaltexten, sondern auch freie Versionen auslieferte: Es handelt sich zwar um „Hinübersetzungen“, doch nicht alle können auf die traditionelle interlinguale Typologie zurückgeführt werden, weil er keine spezifische Äquivalenz zwischen AT und ZT berücksichtigte.

Die meisten seiner Arbeiten fallen unter andere Typologien von Textverarbeitung und werden als „Nachdichtungen“, „Übertragungen“ oder „Bearbeitungen“ bezeichnet. Viele sind eine neue Fassung, eine Art Revision des AT, durch die er den Inhalt des Originals auf eine solche Weise adaptierte und dergestalt verarbeitete, dass sie von ihm sogar neu ausgewertet und umgestaltet wurden. Es sind aber vor allem originalgetreue Übertragungen des Stilmittels, um Neugestaltungen im Sinne nur der Modalität der inhaltlichen Wiedergabe, in welchen der Übersetzer Hildesheimer, statt dem AT beim Wortschatz oder beim Stil wortwörtlich zu folgen, vornehmlich auf die Wirkung des neuen Textes zielte.

Das Anstreben der spontanen Ausdrucksfähigkeit Hildesheimers hat ferner das Gewicht auf einen weiteren Charakterzug von seiner Expressivität gelegt. Wie später der amerikanische Translationswissenschaftler und Übersetzer Lawrence Venuti den Begriff der ,translator's invisibility“ (Venuti 1995) theoretisch entwickelte, vertrat Hildesheimer schon die Idee des ,verschwundenen“ bzw. „unsichtbaren“ Übersetzers, der hinter seiner Arbeit „tatsächlich verschwindet, im Wissen, daß ihm seine Arbeit dann gelungen ist, wenn der Leser weder seine Leistung noch seine Existenz zur Kenntnis nimmt“ (Hildesheimer 1985/1991: 217).

Demgegenüber hat die Analyse beider seiner Reden ein entgegengesetztes Ziel gezeigt, nämlich jenes, die meisten seiner eigenen Termini und Ausdrücke des Originals mit maximaler Genauigkeit, wenn nicht sogar wörtlich, wiederzugeben und sich dadurch vom AT nicht allzu weit zu entfernen. Wörtliche Übersetzungen zeigen nämlich die Intention, nichts vom Autor Gesagten zu verlieren und es vielmehr so wiederzugeben, wie es ursprünglich ausgedrückt wurde. Darüber hinaus verwendete Hildesheimer sowohl beim Englisch- als auch beim Deutschschreiben eine sehr reiche Sprache, die oft Elemente aus anderen Sprachen, wie der französischen, lateinischen und griechischen enthält. Die Analyse der sorgfältig vorbereiteten Wort-für-WortÜbersetzung seiner auf Englisch gehaltenen und ins Deutsche übersetzten Reden hat somit die Anerkennung dessen verstärkt, dass Hildesheimer ein wahrer Sprachliebhaber mit einer dynamischen, einsatzfreudigen Persönlichkeit war, für den der sprachlich-linguistische Aspekt ein integrierender Bestandteil des gegebenen Inhalts war. 


\section{Literaturverzeichnis}

Ammann, Margret (1990): „Rezension von Albrecht u. a. (ed.) (1987): Translation und interkulturelle Kommunikation.“ TextConText. Translation, Theorie, Didaktik, Praxis 9/1990: 106-129.

Anselmi, Simona (2012): On Self-Translation: An Exploration in Self-translators' Teloi and Strategies. Milano: LED.

Bhatia, Tej K./Ritchie, William C. (ed.) (2004): The Handbook of Bilingualism. Malden/MA: Blackwell.

Braese, Stephan (2016): Jenseits der Pässe: Wolfgang Hildesheimer. Eine Biographie. Göttingen: Wallstein.

Broeck, Raymond van den (1981): „The Limits of Translatability Exemplified by Metaphor Translation“. In: Even-Zohar, Itamar/Toury, Gideon (eds.): Translation theory and intercultural relations [= Poetics Today 2/4]. Tel Aviv: The Porter Institute for Poetics and Semiotics, Tel Aviv University: 73-87. doi: 10.2307/1772487.

Bußmann, Hadumod (ed.) (2008): Lexikon der Sprachwissenschaft. 4., durchgesehene und bibliographisch ergänzte Auflage unter Mitarbeit von Hartmut Lauffer. Stuttgart: Kröner.

Cedillo, Ana Caro (2004): Fachsprachliche Kollokationen. Ein übersetzungsorientiertes Datenbankmodell Deutsch-Spanisch. Tübingen: Narr.

Chesterman, Andrew (2009): „The Name and Nature of Translator Studies“. Hermes - Journal of Language and Communication Studies 42: 13-22.

Chung, Gou-Yeun (2013): „Ipsoübersetzung: Untersuchung der Funktionsgerechtigkeit von Abweichungen in Ahn Junghyos Selbstübersetzung Silver Stallion“. 독일언어문학 제62집: KOAJ/Korea Open Access Journals 62: 95-113. doi: 10.30947/zfds1.2013.62.95.

Collins Cobuild (1987), English Language Dictionary. Collins Birmingham University International Language Database, London/Glasgow: Collins/Stuttgart: Klett.

Collins Online English Dictionary (2020). collinsdictionary.com [29.12.2020].

Duden (2016): Die Grammatik. Unentbehrlich für richtiges Deutsch. Wöllstein, Angelika/Duden-Redaktion (ed.). 9., vollständig überarbeitete und aktualisierte Auflage. Berlin: Dudenverlag.

Duden (2014): Das Synonymwörterbuch. Ein Wörterbuch sinnverwandter Wörter. 6., vollständig überarbeitete Auflage. Dudenredaktion (ed.). Bd. 8. Berlin: Dudenverlag.

Duden (2013): Redewendungen. Wörterbuch der deutschen Idiomatik. Bd. 11. Berlin: Bibliographisches Institut.

Duden (2011): Deutsches Universalwörterbuch. Dudenredaktion (ed.). 7., überarbeitete und erweiterte Auflage. Mannheim/Zürich: Dudenredaktion.

Duden (2003): Deutsches Universalwörterbuch. Dudenredaktion (ed.). 5., überarbeitete Auflage. Mannheim etc.: Dudenverlag.

Duden (2001): Das Herkunftswörterbuch: Etymologie der deutschen Sprache. Die Geschichte der deutschen Wörter bis zur Gegenwart. Bd. 7. Mannheim u. a.: Dudenverlag.

Duden (1997): Die sinn- und sachverwandten Wörter. Bd. 8. Mannheim etc.: Bibliographisches Institut.

Eichinger, Ludwig M. (2000): Deutsche Wortbildung. Eine Einführung. Tübingen: Narr. 
Grutman, Rainier (2009): "Self-Translation". In: Baker, Mona/Saldanha, Gabriela (eds.): Routledge Encyclopedia of Translation Studies. London/New York, Routledge: 257-260.

Grutman, Rainier (2001): „Auto-translation“. In: Baker, Mona (ed.): Routledge Encyclopedia of Translation Studies. London/New York, Routledge: 17-20.

Hammer, Maud (1999): Zweisprachige Kindererziehung. Diplomarbeit zur Erlangung des akademischen Grades einer Magistra der Philosophie. Graz: Karl-Franzens-Universität.

Hausmann, Albrecht (2005): „Übertragungen: Vorüberlegungen zu einer Kulturgeschichte des Reproduzierens". In: Bußmann, Britta et al. (eds.): Übertragungen. Formen und Konzepte von Reproduktion in Mittelalter und Früher Neuzeit. Berlin/New York, de Gruyter: XI-XX.

Hildesheimer, Wolfgang (1999): Briefe. Hildesheimer, Silvia/Pleyer, Dietmar (eds.). Frankfurt a. M.: Suhrkamp.

Hildesheimer, Wolfgang (1993): Ich werde nun schweigen. Gespräch mit Hans Helmut Hillrichs in der Reihe „Zeugen des Jahrhunderts “. Herausgegeben von Ingo Hermann. Göttingen: Lamuv.

Hildesheimer, Wolfgang (1985/1991): „Der Autor als Übersetzer. Der übersetzte Autor“. In: Ders.: Gesammelte Werke in sieben Bänden. Herausgegeben von Hart Nibbrig, Christiaan Lucas und Volker Jehle. Bd. VII: Vermischte Schriften. Frankfurt a. M., Suhrkamp: 211217.

Hildesheimer, Wolfgang (1984/1991a): „The Jewishness of Mr. Bloom. ,Bloomsday Dinner Speech““. In: Ders.: Gesammelte Werke in sieben Bänden. Herausgegeben von Hart Nibbrig, Christiaan Lucas und Volker Jehle. Bd. VII: Vermischte Schriften. Frankfurt a. M., Suhrkamp: 183-195.

Hildesheimer, Wolfgang (1984/1991b): „Das Jüdische an Mr. Bloom“. In: Ders.: Gesammelte Werke in sieben Bänden. Herausgegeben von Hart Nibbrig, Christiaan Lucas und Volker Jehle. Bd. VII: Vermischte Schriften. Frankfurt a. M., Suhrkamp: 196-210.

Hildesheimer, Wolfgang (1975/1991a): „The End of Fiction“. In: Ders.: Gesammelte Werke in sieben Bänden. Herausgegeben von Hart Nibbrig, Christiaan Lucas und Volker Jehle. Bd. VII: Vermischte Schriften. Frankfurt a. M., Suhrkamp: 125-140.

Hildesheimer, Wolfgang(1975/1991b): „Das Ende der Fiktionen”. In: Ders.: Gesammelte Werke in sieben Bänden. Herausgegeben von Hart Nibbrig, Christiaan Lucas und Volker Jehle. Bd. VII: Vermischte Schriften. Frankfurt a. M., Suhrkamp: 141-158.

Hildesheimer, Wolfgang (1969/1991): „Übersetzung und Interpretation einer Passage aus ,Finnegans Wake“ von James Joyce“. In: Ders.: Gesammelte Werke in sieben Bänden. Herausgegeben von Hart Nibbrig, Christiaan Lucas und Volker Jehle. Bd. VII: Vermischte Schriften. Frankfurt a. M., Suhrkamp: 338-351.

Hokenson, Jan/Munson, Marcella (2007): The Bilingual Text. History and Theory of Literary Self-Translation. Manchester/Kinderhook: St. Jerome Publishing.

Ivir, Vladimir (2004): „Descriptive and Prescriptive Approaches in the Linguistic Study of Translation“. In: Kittel, Harald et al. (eds.): Übersetzung - Translation - Traduction. Ein internationales Handbuch zur Übersetzungsforschung/An International Encyclopedia of Translation Studies. Bd. 1. Berlin, Walter de Gruyter: 276-284.

Jakobson, Roman (1959): „On Linguistic Aspects of Translation“. In: Brower, Reuben A. (ed.):

On Translation. Cambridge/Massachusetts, Harvard University Press: 232-239. 
Jehle, Volker (2016): „,Die sichtbare Wirklichkeit bedeutet mir nichts “. Die Briefe an die Eltern. Mit zahlreichen Abbildungen. Jehle, Volker (ed.). 2 Bd. Berlin: Suhrkamp Verlag.

Jehle, Volker (2003): Wolfgang Hildesheimer - Werkgeschichte. Bd. 2. Nordhausen: Traugott Bautz Verlag.

Koller, Werner (2011): Einführung in die Übersetzungswissenschaft. 8., neubearbeitete Auflage. Tübingen: Narr Francke Verlag.

Kromann, Hans-Peder (1989): „Zur funktionalen Beschreibung von Kollokationen und Phraseologismen in Übersetzungswörterbüchern“. In: Gréciano, Gertrud (ed.): Europhras 88. Phraséologie contrastive. Actes du Colloque International de Phraséologie Contrastive. Klingenthal-Strasbourg 12-16 mai 1988. Strasbourg, Université des sciences humaines, Département d'études allemandes: 265-272.

Kromann, Hans Peder/Rieber, Theis/Rosbach, Paul (1984): „Überlegungen zu Grundfragen der zweisprachigen Lexikographie“. In: Wiegand, Herbert Ernst (ed.): Studien zur neuhochdeutschen Lexikographie V. Hildesheim, Olms: 159-238. (= Germanistische Linguistik 36/1984).

Lausberg, Heinrich (1960/1990): Handbuch der literarischen Rhetorik: eine Grundlegung der Literaturwissenschaft. 3., Auflage. Stuttgart: Steiner.

Loserl, Christine (2011): Übersetzung und Selbstübersetzung im Werk von Ariel Dorfman. Diplomarbeit, Universität Wien.

Mackensen, Lutz (2013): Ursprung der Wörter. Das etymologische Wörterbuch der deutschen Sprache. Vollständig überarbeitete und aktualisierte Neuausgabe des berühmten Herkunftswörterbuch. München: Bassermann.

Nickel, Gerhard (1980): „Kontrastive Linguistik“. In: Althaus, Hans Peter et al. (eds.), Lexikon der Germanistischen Linguistik. 2., Auflage. Tübingen, Niemeyer: 633-636.

Online Etymology Dictionary. etymonline.com [29.12.2020].

Routledge Encyclopedia of Translation Studies (1998/2001): Baker, Mona (ed.). London/New York: Routledge.

Salmon, Laura (2013): „Il processo autotraduttivo: definizioni e concetti in chiave epistemologico-cognitiva“" In: Ceccherelli, Andrea et al. (eds.): Autotraduzione e riscrittura. Bologna, Bononia University Press: 77-98.

Salvato, Lucia (2018): „Dalla Traduzione all'autotraduzione. Quali cambiamenti linguistici comporta la ,divulgazione culturale"?". In: Calzoni, Raul (ed.), La circolazione del sapere nei processi traduttivi della lingua letteraria tedesca. Il Quadrifoglio tedesco 35, Milano/Udine, Mimesis edizioni: 61-78.

Salvato, Lucia (2019): Scelte linguistiche e strategie comunicative nell'autotraduzione tedesca: Ruth Klüger e Wolfgang Hildesheimer a confronto. In: Berni, Bruno/D’Atena, Alessandra (eds.), Autotraduzione. Obiettivi, strategie, testi. Roma, Istituto Italiano Studi Germanici: 95-121.

Sapir, Edward (1921): Language. An Introduction to the Study of Speech. New York: Harcourt Brace.

Sapir, Edward (1983): Selected Writings of Edward Sapir in Language, Culture, and Personality. Ed. by David G. Mandelbaum. Berkeley: University of California Press.

Schreiber, Michael (1993): Übersetzung und Bearbeitung. Zur Differenzierung und Abgrenzung des Übersetzungsbegriffs. Tübingen: Narr. 
Schreiber, Michael (2004): „Übersetzung und andere Formen der Textverarbeitung und Textreproduktion in sprachwissenschaftlicher Sicht“. In: Frank, Armin Paul et al. (eds.): Übersetzung - Translation - Traduction. Ein internationales Handbuch zur Übersetzungsforschung. An international Encyclopedia of Translation Studies. Encyclopédie internationale des sciences de traduction. 1. Teilband. Berlin/New York, de Gruyter: 268-275.

Toury, Gideon (2012): Descriptive Translation Studies and Beyond. Revised edition. Amsterdam/Philadelphia: Benjamins.

Trier, Jost (1931): Der deutsche Wortschatz im Sinnbezirk des Verstandes. Die Geschichte eines sprachlichen Feldes. 1: Von den Anfängen bis zum Beginn des 13. Jahrhunderts. Heidelberg: Winter.

Venuti, Lawrence (1995): The Translator's Invisibility. A History of Translation. London/New York: Routledge.

Weinrich, Harald (2007), Textgrammatik der deutschen Sprache, unter Mitarbeit von Thurmair, Maria et al. 3., revidierte Auflage. Darmstadt: Wiss. Buchges.

Weinrich, Harald (1980): „Metapher". In: Ritter, Joachim et al. (eds.) Historisches Wörterbuch der Philosophie. Völlig neubearb. Ausg. des Wörterbuchs der philosophischen Begriffe von Rudolf Eisler. Bd. 5. Basel etc., Schwabe: 1179-1186.

Weisgerber, Leo (1971): Grundzüge der inhaltsbezogenen Grammatik. 4., Auflage. Düsseldorf: Schwann. (= Von den Kräften der deutschen Sprache I).

Whorf, Benjamin Lee (1956): Language, Thought, and Reality: Selected Writings of Benjamin Lee Whorf. Cambridge/Mass. (dt. Teilübersetzung: Sprache, Denken, Wirklichkeit. Beiträge zur Metalinguistik und Sprachphilosophie. Hrsg. und übers. von Peter Krausser. Rowohlt: Reinbeck bei Hamburg 1963).

Wilpert, Gero von (2001): Sachwörterbuch der Literatur. 8., erweiterte Auflage. Stuttgart: Alfred Kröner Verlag.

Winhart, Heike (2005): Funktionsverbgefüge im Deutschen. Zur Verbindung von Verben und Nominalisierungen. Dissertation, Neuphilologisch Fakultät der Universität Tübingen. publikationen.uni-tuebingen.de/xmlui/bitstream/handle/10900/46248/pdf/DissertationDrive.pdf? sequence=1\&isAllowed=y. [29.12.2020]. 